\title{
Extra-solar Planets: Clues to Planetary Formation
}

\author{
Nuno C. Santos \\ Observatório Astronómico de Lisboa/CAAUL, Tapada da Ajuda, \\ 1349-018 Lisboa, Portugal and Observatoire de Genève, 1290 Sauverny, \\ Switzerland
}

Michel Mayor, Dominique Naef, Francesco Pepe, Didier Queloz, and Stéphane Udry

Observatoire de Genève, 1290 Sauverny, Switzerland

\begin{abstract}
Radial velocity surveys have revealed up to now about 115 extra-solar planets, among which a few multi-planetary systems. The discovered planets present a wide variety of orbital elements and masses, which are raising many problems and questions regarding the processes involved in their formation. The statistical analysis of the distributions of orbital elements, planetary masses, and relations between these, is however already giving some strong constraints on the formation of the planetary systems. Furthermore, the study of the planet host stars has revealed the crucial role of the stellar metallicity on the giant planet formation. In this paper we will review the current status of the research on this subject.
\end{abstract}

\section{Introduction}

Following the discovery of a giant planet orbiting the solar-type star 51 Peg (Mayor \& Queloz 1995), planet hunters have unveiled the presence about 115 exo-worlds ${ }^{1}$. These discoveries, that include $\sim 10$ multi-planetary systems (e.g. Butler et al. 1999; Mayor et al. 2003b), and one confirmed transiting planet (Charbonneau et al. 2000; Henry et al. 2000), have brought to light the existence of planets with a huge variety of characteristics, opening unexpected questions about the processes of giant planet formation. The definition of a planet has itself been put into question.

But with the numbers increasing very fast (see Mayor et al. 2003a; 2003b, for the latest announcement), current results are already giving us the chance to undertake the first statistical studies of the properties of the exo-planets, as well

\footnotetext{
${ }^{1}$ Counts on September 2003; see table at http://obswww.unige.ch/exoplanets for continuous updates. Before these discoveries, only planets around a pulsar had been detected (Wolszczan \& Frail 1992); these are probably second generation planets, however. Also, the previously discovered radial-velocity companion around HD 114762 (Latham et al. 1989) has a minimum mass above $10 \mathrm{M}_{J u p}$, and a very low $[\mathrm{Fe} / \mathrm{H}]$ (Santos et al. 2003a), being thus most likely a brown-dwarf
} 

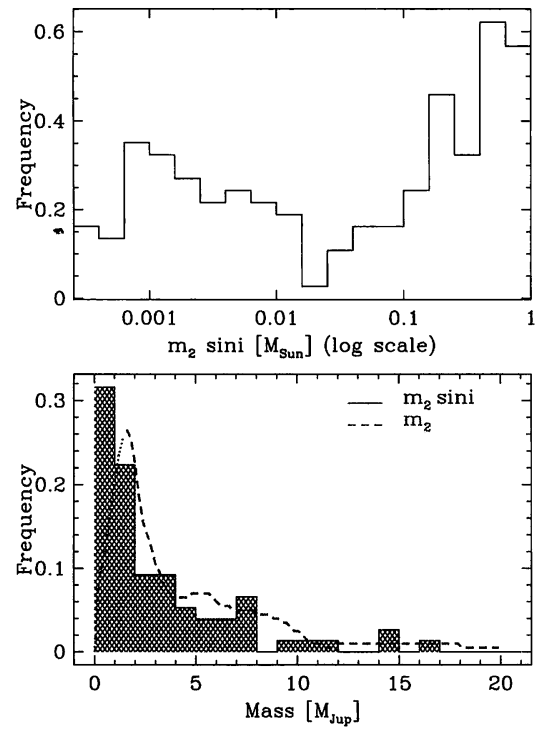

Figure 1. Mass function of companions to solar-type stars in log (top) and linear (bottom) scales. In the lower panel, the dashed line represents the result of a statistical deconvolution of the observed distribution in order to take into account the effect of the orbital inclination. As in Jorissen et al. (2001)

of their host stars. This is bringing new interesting constraints for the models of planet formation and evolution. In the rest of this article we will review these results, focusing on the various observational constraints the new discoveries are bringing. Most of the results presented here have also been published (or are currently in preparation) in a series of papers devoted to the study and analysis of the statistical properties of exoplanets (Udry, Mayor, \& Santos 2003; Santos et al. 2003a; Eggenberger, Mayor, \& Udry 2003; and other articles in the series).

\section{Clues from the planetary parameters}

\subsection{The Mass distribution}

One important clue concerning the nature of the now discovered planetary systems comes from their mass distribution (Fig. 1).

Several conclusions may be taken from the plots. First, a look at the upper panel of Fig. 1, shows that there is a clear gap in the mass distribution of the companions to solar-type stars. This gap, separating low mass stellar companions from the planetary-mass objects (often called the "brown dwarf desert") represents a strong evidence that these two populations are the result of different formation and/or evolution processes. 
A zoom-up of the low-mass part of this plot (lower panel of Fig. 1) also tells us something very interesting. We can see here that although the radial-velocity technique is more sensitive to more massive companions, the planetary mass distribution rises towards the low mass regime. Furthermore, the distribution drops to zero at masses around $\sim 10 \mathrm{M}_{\text {Jup }}$ (Jorissen, Mayor, \& Udry 2001), although the tail of the distribution may extend up to a mass of $\sim 20 \mathrm{M}_{J u p}$. This limit is not related to the Deuterium-burning mass limit of $\sim 13 \mathrm{M}_{\text {Jup }}$, sometimes considered as the arbitrary limiting mass for a planet ${ }^{2}$. As it was recently shown by Jorissen et al. (2001), this result is not an artifact of the fact that for most of the targets we only have minimum masses, but a real upper limit for the mass of the planetary companions discovered so far, since it is clearly visible in a deconvolved distribution, where the effect of the unknown orbital inclination was taken into account.

\subsection{Orbital Period}

One of the most interesting problems that appeared after the first planets were discovered has to do with the proximity to their host stars. In contrast with the current observations, giant planets were previously thought to form (and be present) only at distances of a few A.U. from their "suns" (Pollack et al. 1996). However, and in striking contrast with the predictions, the first exoplanets were found very close to their parent stars. This result has led to a change in the paradigm of planetary formation and evolution. To explain the new systems, it is now clear that the theories have to include orbital migration due to interactions with the gas disk (Goldreich \& Tremaine 1980; Ward 1997).

Although still quite biased for the long period systems (more difficult to detect by the radial-velocity surveys), the period distribution of the extra-solar planetary companions can already tell us something about the planetary formation and evolution processes. This is particularly true for the short period systems, for which the biases are not so important. In particular, one of the most impressive features present in the current data is the clear pile-up of planetary companions with periods $\sim 3$ days (although a planet with a period of 2.5 days has been recently detected - Udry et al. 2003b), and the absence of any system with a period shorter than this ${ }^{3}$. This result, that is in complete contrast with the period distribution for stellar companions ${ }^{4}$ (e.g. Mayor \& Santos 2002), means that somehow the process involved in the planetary migration makes the planet "stop" at a distance corresponding to this orbital period. To explain this fact, several ideas have been presented, invoking e.g. a magnetospheric central cavity of the accretion disk, tidal interaction with the host star, Roche-lobe overflow by the young inflated giant planet, or evaporation.

\footnotetext{
${ }^{2}$ This value is an arbitrary limit used as a "working definition", but it is not related to the planetary formation physics

${ }^{3} \mathrm{~A}$ few transiting candidates have been suggested to have periods significantly shorter than this value (e.g. Konacki et al. 2002), but the true nature of the signals is still under debate

${ }^{4}$ Stellar binaries are not limited to periods longer than this limit, even when the mass of the secondary is in the brown-dwarf domain - e.g. HD 41004Bb with $\mathrm{P}=1.3$ days (Santos et al. 2002)
} 


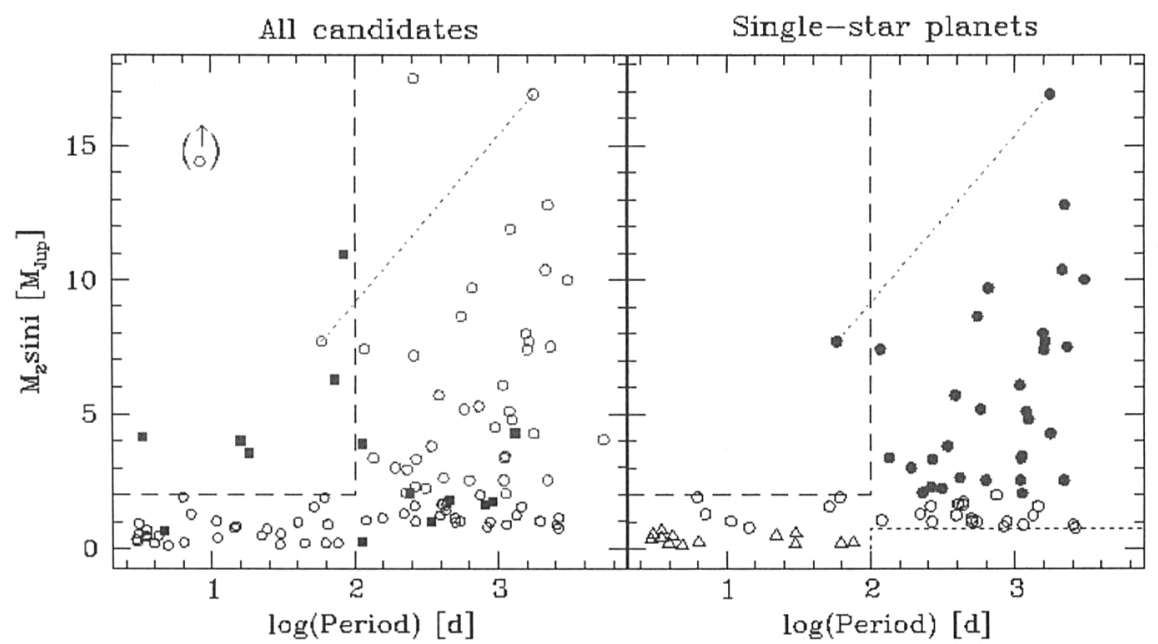

Figure 2. Minimum masses versus periods for known exoplanet candidates. In the left panel, filled squares indicate planets in binaries whereas circles are used for planets around single stars. In the right panel, only planets orbiting single dwarf stars are represented. A different coding is used for massive $\left(m_{2} \sin i \geq 2 \mathrm{M}_{\mathrm{Jup}}\right.$; filled symbols), intermediate-mass ( $m_{2} \sin i$ between 0.75 and $2 \mathrm{M}_{\mathrm{Jup}}$; open circles), and lighter $\left(m_{2} \sin i \leq 0.75 \mathrm{M}_{\text {Jup }}\right.$; open triangles) candidates. The dashed and dotted lines in the panels indicate limits at $P=100 \mathrm{~d}$ (vertical), at $m_{2} \sin i=2 \mathrm{M}_{\mathrm{Jup}}$ (horizontal left), or at $m_{2} \sin i=0.75 \mathrm{M}_{\text {Jup }}$ (horizontal right). See Udry et al. (2003a) for more details.

\subsection{The Mass-Period relation}

A lot of constraints for the migration scenarii are now being put forward by the analysis of the mass-period relation. In fact, recent results have shown that there seems to be a strong relation between the mass and orbital period of the giant planets. Indeed, a look at Fig. 2 (where we plot these two quantities) reveals a paucity of high-mass planetary companions $\left(\mathrm{M}>2 \mathrm{M}_{J u p}\right)$ orbiting in short period (lower than 100-days) trajectories (Zucker \& Mazeh 2002; Udry et al. 2003a). This trend, clearly significant ${ }^{5}$, is less evident for those planets orbiting stars that have other stellar companions, showing that planet formation (and/or evolution) might be influenced in these systems (e.g. Eggenberger et al. 2003). But overall, these results are indeed compatible with the current ideas about planetary orbital migration (either due to an interaction with the disk or with other companions) - (e.g. Trilling, Lunine, \& Benz 2002) - that teach us that the higher mass planets should migrate less.

Curiously, on the other side of the distribution, there also seems to be a paucity of very low mass giant planets orbiting in long period orbits (Udry et

\footnotetext{
${ }^{5}$ These planets are the easiest to find using radial-velocity instruments
} 

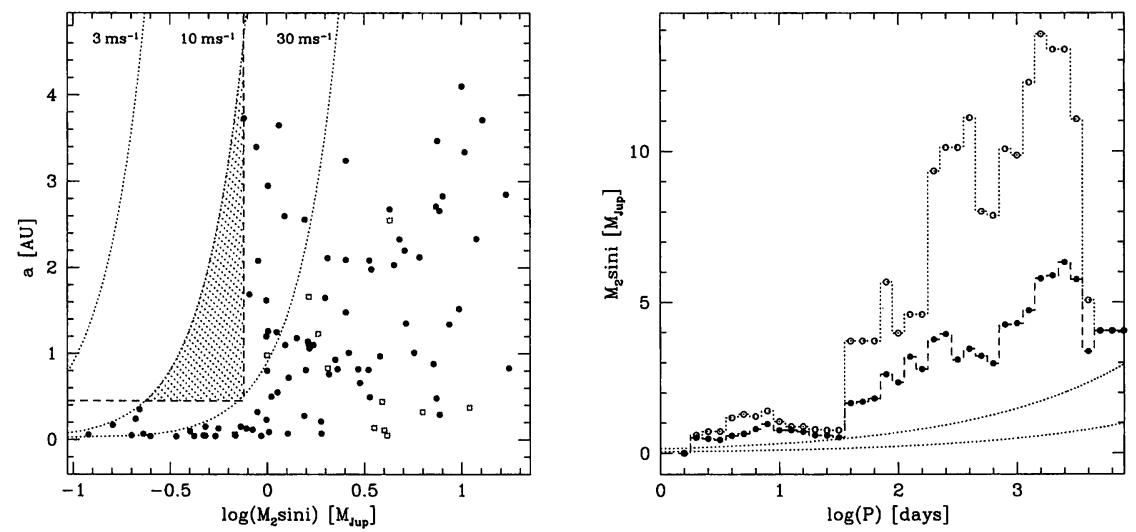

Figure 3. Left panel: Mass-separation diagram for the known exoplanet candidates. The dotted lines illustrate the radial-velocity semiamplitude expected on a solar-mass star due to planets on circular orbits with given minimum masses and separations. The shaded area empty of planets is shown not to be due to small number statistics. Planets in binaries are indicated by open symbols. Right panel: Mean mass (filled circles), or higher mass (average on the 3 higher values; open circles) of planets in period smoothing windows with $\log P=0.2$. From Udry et al. (2003a)

al. 2003a) - Fig. 3, left panel. Actually, all planets with mass lower than about $0.75 \mathrm{Jup}$ are found at close distances from their stars. And although such a trend could be expected from biases related to the radial-velocity surveys, montecarlo simulations have shown that this result is indeed statistically significant. Furthermore, it seems that from the theoretical point of view, this observations might be explained in a scenario of runaway migration, a phenomenon that seems to be very dependent on the mass of the planet (Masset \& Papaloizou 2003).

In other words, low mass planets seem to migrate very fast, while their high-mass counterparts do not migrate significantly from their initial positions. The higher the mass of a planet, the less it will migrate (see also right panel of Fig. 3). One of the consequences of this is the low number of planets at intermediate periods (Udry et al. 2003a), forming the now called period-valley. It should be noted, however, that this "rule" cannot be extrapolated to e.g. much lower mass planets or planets formed at very large distances from the star (e.g. Uranus and Neptune).

Together with these findings, it has recently been suggested that there might be a relation between mass ratio and period ratio for planets in multiple systems (Mazeh \& Zucker 2003). If confirmed, this trend may also be telling us something more about the formation and evolution of multi-planetary systems. 


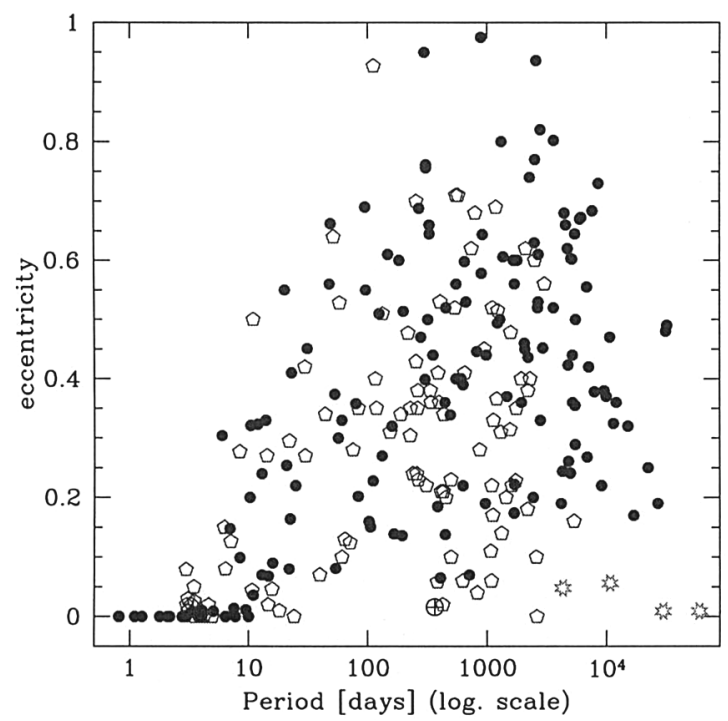

Figure 4. The $e-\log P$ diagram for planetary (open pentagons) and stellar companions (filled circles) to solar type field dwarfs. Starred symbols represent the giant planets of our Solar System, while the "Earth" symbol represents our planet

\subsection{The eccentricity}

One of the most enigmatic results to date is well illustrated in Fig. 4. A first look at the figure shows that there are no clear differences between the eccentricity distributions of planetary and stellar binary systems. How can two groups of bodies, formed by physically different processes, have basically the same distribution in this plot? And how then can this be fit into the "traditional" picture of a planet forming in a disk? Actually, for masses lower than $\sim 20 M_{J u p}$, it has been suggested that the interaction (and migration) of a companion within a gas disk may have the effect of damping its eccentricity (Goldreich \& Tremaine 1980; Ward 1997). This implies that other processes may play an important role in defining the "final" orbital configuration. Possible candidates include the interaction between planets in a multiple system (e.g. Rasio \& Ford 1996) or between the planet and a disk of planetesimals (e.g. Murray et al. 1998), the simultaneous migration of various planets in a disk (e.g. Murray, Paskiwitz, \& Holman 2002), the influence of a distant stellar companion (e.g. Mazeh \& Shaham 1979, Cochran et al. 1997), or even the interaction with the gaseous disk itself (Goldreich \& Sari 2003). In this respect, one particularly interesting case of very high eccentricity (above 0.9 ) amongst the planetary companions is the planet around HD 80606 (Naef et al. 2001).

Although still not clear, however, a close inspection of Fig. 4 permits to find a few differences between the eccentricities of the stellar and planetary companions (Mayor \& Udry 2000; Halbwachs, Mayor, \& Udry 2003). For example, for periods in the range of 10 to 30 days, clearly outside the circularization period by 

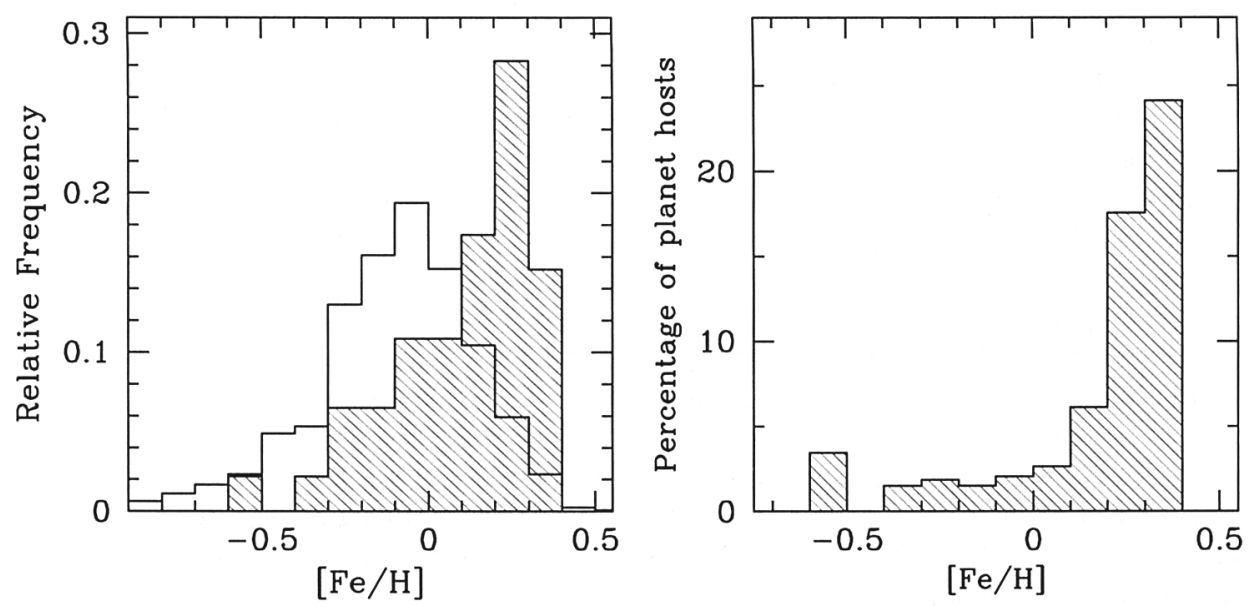

Figure 5. Left: metallicity distribution of stars with planets making part of the CORALIE planet search sample (shaded histogram) compared with the same distribution for the about 1000 non binary stars in the CORALIE volume-limited sample. Right: the result of correcting the planet hosts distribution to take into account the sampling effects. The vertical axis represents the percentage of planet hosts with respect to the total CORALIE sample. From Santos et al. (2003a).

tidal interaction with the star, there are a few stars with planets having very low eccentricity, while no stellar binaries are present in this region. The same and even more conspicuous trend is seen for longer periods, suggesting the presence of a group of planetary companions with orbital characteristics more similar to those of the planets in the Solar System. On the other hand, for the very short period systems, we can see some planetary companions with eccentricities higher than those found for stellar companions of similar periods. These facts may be telling us that different formation and/or evolution processes took place: for example, the former group may be seen as a sign of formation (and evolution) in a disk, and the latter one as an evidence of the gravitational influence of a longer period companion on the eccentricity.

\section{The metal-rich nature of planet host stars}

Up to now we have been reviewing the results and conclusions we have obtained directly from the study of the orbital properties and masses of the discovered planets. But another particular fact that is helping us to understand the mechanisms of planetary formation has to do with the planet host stars themselves. In fact, they were found to be particularly metal-rich, i.e. they have, in average, a metal content higher than the one found in stars without detected planetary companions (e.g. Gonzalez 1998; Gonzalez et al. 2001; Santos, Israelian, \& Mayor 2001; Santos et al. 2003a). This result, clearly confirmed by an uniform spectroscopic analysis of large samples of stars with and without detected giant planets (Santos et al. 2001), was further shown not to be due to any sampling or 
observational biases (e.g. Santos et al. 2003a), and is obtained by using different kinds of techniques to derive the stellar metallicity ${ }^{6}$.

The most recent studies seem to favor that this metallicity "excess" is original from the cloud that gave origin to the star/planetary system (Pinsonneault, DePoy, \& Coffee 2001; Santos et al. 2001, 2003a; Sadakane et al. 2002), and not a result of the engulfment of planetary (iron rich) material into the stellar convective envelopes ${ }^{7}$. Furthermore, they show that the probability of finding a planet is proportional to the metallicity of the star: more metal-rich stars have a higher probability of harboring a planet than lower metallicity objects (Santos et al. 2001, 2003a; Reid 2002; Laws et al. 2003) - Fig. 5, right panel ${ }^{8}$.

A possible and likely interpretation of this is saying that the higher the metallicity of the cloud that gives origin to the star/planetary system (and thus the dust content of the disk), the faster a planetesimal can grow, and the higher the probability that a giant planet is formed before the proto-planetary disk dissipates. In other words, the metallicity seems to be playing a key role in the formation of the currently discovered extra-solar planetary systems (see e.g. discussion in Santos et al. 2003a for further details).

These conclusions have many implications for the theories of planetary formation. In this respect, two main cases are now debated in the literature. On the one side, the traditional core accretion scenario (e.g. Pollack et al. 1996) tells us that giant planets are formed as the result of a runaway accretion of gas around a previously formed icy core with about 10 times the mass of the Earth. Opposite to this idea, Boss (1997) (see also Mayer et al. 2002) has proposed that giant planets may form by a disk instability process. The main advantage of this model is the shorter timescales needed to form planets: although not clear, in the traditional core-accretion model, the formation of a giant planet may take longer than the currently estimated lifetimes of T-Tauri disks (e.g. Haisch et al. 2001); recent developments have, however, put new constraints into the disk lifetimes, that may be considerably longer than previously predicted (Bary, Weintraub, \& Kastner 2003). However, according to the instability model, the efficiency of planetary formation should not be dependent on the metallicity of the star/disk (Boss 2002). The results presented above, showing that the probability of finding a planet is a strong function of the stellar metallicity, thus favor the former (core-accretion) model as the main mechanisms responsible for the formation of giant planets (although they do not completely exclude the disk instability model).

It should be cautioned, however, that it is not known precisely how the influence of the metallicity is influencing the planetary formation and/or evolution; for example, the mass of the disks themselves, that can be crucial to

\footnotetext{
${ }^{6}$ In most studies, the iron abundance, or $[\mathrm{Fe} / \mathrm{H}]$, is used as the stellar metallicity index. Studies on the abundances of other elements on planet host stars are reviewed by G. Israelian in this volume

${ }^{7}$ There are, however, some hints of stellar pollution, but not necessarily capable of changing significantly the global metal-content of the star (e.g. Israelian et al. 2001, 2003; Laws \& Gonzalez 2001; Gratton et al. 2001); see also contribution by G. Israelian in this volume
}

${ }^{8} \mathrm{~A}$ confirmation of this results has also been presented in this meeting by D. Fischer 
determine the efficiency of planetary formation, is not known observationally with enough precision.

\section{Black Sheep}

The non-conventional properties of many of the exoplanet candidates raised at first some skepticism from the community. The first exoplanet discovered, orbiting the solar-type star 51 Peg (Mayor \& Queloz 1995), is itself a good example. Its particularly short-period orbit ( $\sim 4.23$ days) led some astronomers to cast doubts about its existence. For example, Gray (1997) suggested that the radialvelocity variations were due to non-radial pulsations rather than to the presence of a planetary mass companion. Later on, this result was withdrawn (Gray 1998) and the presence of the planet around 51 Peg confirmed.

Other similar examples exist in the literature. Exploring the fact that the radial-velocity technique only gives us the minimum mass for the companion, Han, Black, \& Gatewood (2001) suggested that the planetary candidates were in fact low mass stars on orbits seen edge-on. This result was, however, easily refuted by several recent works (Halbwachs et al. 2000; Jorissen et al. 2001; Pourbaix 2001; Pourbaix \& Arenou 2001). Again, the "planetary origin" of the radial-velocity variations was then considered to be the best one.

It is well known that intrinsic stellar features, like non-radial pulsation, inhomogeneous convection, or spots may induce radial-velocity variations (Brown et al. 1998; Saar \& Donahue 1997; Saar, Butler, \& Marcy 1998; Santos et al. 2000b; Paulson et al. 2002; Tinney et al. 2002). These situations can prevent us from finding planets, if the perturbation is larger than the orbital radial-velocity variation, or even give us false candidates, if they produce a periodic signal over a few rotational periods. In this sense, it is interesting to note that at this meeting, S.V. Berdyugina has presented evidences for persistent star-spots in the Sun and possibly in other stars. A good example of this effect is the periodic radial-velocity signal observed for the dwarf HD 166435, that was shown to be due to a spot rather than to the presence of a planet (Queloz et al. 2001).

The presence of unknown stellar blends can also induce spurious radialvelocity signals, which can "simulate" the presence of a planetary companion in the case of triple systems. An example is given by HD 41004 in which the moving spectrum of a faint spectroscopic binary companion induces a planetarytype signature on the primary star (Santos et al. 2002; Zucker et al. 2003).

In this context, another planetary companion that was recently called back into question is the case of HD 192263. This star was announced to harbor a Jupiter-mass planetary companion on a $\sim 24$-day period orbit (Santos et al. 2000a; Vogt et al. 2000). Recently however, Henry, Donahue, \& Baliunas (2002) have detected a photometric variation with a period compatible with the period observed in the radial-velocity data. The authors have then suggested that the planet around this star was no longer needed to explain the radial-velocity signal. Once again, however, the doubts about the planetary origin of the radial-velocity variations of HD 192263 seem to have disappeared, since it has been shown that the observed (sporadic) photometric variations are not correlated at all with the radial-velocity signal. This latter shows an extremely constant period, phase, and amplitude over the last 4 years (Santos et al. 2003b). 
This series of cases show that the planetary nature of most of the systems discovered so far is extremely well established. Although it is conceivable that some flaws exist, globally the planetary companions now discovered are indeed planets.

\section{Concluding Remarks}

The study of extra-solar planetary systems is giving its first steps. After only 8 years, we can say that at least $5 \%$ of the solar type dwarfs have giant planetary companions, with masses as low as the mass of Saturn and orbital separations of a few $\mathrm{AU}$ (the limits imposed by the current planetary search techniques).

As we have seen above, the observed correlations between the orbital parameters of the newly found planets are giving astronomers a completely different view on the processes of formation and evolution of the planetary systems. As the numbers increase, the first statistically significant studies give us the opportunity to revise the theories. Slowly we are building a new picture.

Furthermore, the analysis of the chemical properties of the planet host stars is giving us a lot of interesting information. These latter studies have revealed the crucial role the metallicity is playing into the formation of the currently found planetary systems, showing that the percentage of stars harboring giant planets is a strongly rising function of the stellar metallicity.

As the planet search programs continue their way, many more planetary companions are expected to be discovered in the next few years. In particular, many hopes are now coming from state-of-the-art spectrographs like HARPS (Pepe et al. 2002), capable of achieving the $1 \mathrm{~m} / \mathrm{s}$ precision (Mayor et al. 2003a). This will give us the opportunity to improve the statistical analysis, and to better understand the physics beyond the formation of the planetary systems.

Acknowledgments. We would like to thank G. Israelian, and R. Rebolo, who have largely contributed to the results presented here. We wish to thank the Swiss National Science Foundation (Swiss NSF) for the continuous support to this project. N.C.S. thanks the support of the European Commission through the The Formation and Evolution of Young Stellar Clusters Research Training Network, as well as of the Fundação para a Ciência e Tecnologia, Portugal, in the form of a scholarship.

\section{References}

Bary, J.S., Weintraub, D.A., \& Kastner, J.H. 2003, ApJ, 586, 1136

Boss, A. 2002, ApJ, 567, L149

Boss, A. 1997, Science, 276, 1836

Brown T.M., Kotak R., Horner S., Kennelly, E.J., Korzennik, S., Nisenson, P., \& Noyes, R.W. 1998, ApJS 117, 563

Butler, R.P., Marcy, G.W., Fischer, D.A., Brown, T.M., Contos, A.R., Korzennik, S.G., Nisenson, P., \& Noyes, R.W. 1999, ApJ, 526, 916

Charbonneau D., Brown T.M., Latham D.W., \& Mayor M. 2000, ApJ, 529, L45

Cochran, W.D., Hatzes, A.P., Butler, R.P., \& Marcy, G.W. 1997, ApJ, 483, 457 
Eggenberger, A., Mayor, M., \& Udry, S. 2003, A\&A, in press

Goldreich, P., \& Sari, R. 2003, ApJ, 585, 1024

Goldreich, P., \& Tremaine, S. 1980, ApJ, 241, 425

Gonzalez G., Laws C., Tyagi S., \& Reddy B.E. 2001, AJ121, 432

Gonzalez, G. 1998, A\&A, 334, 221

Gratton, R.G., Bonanno, G., Claudi, R.U., Cosentino, R., Desidera, S., Lucatello, S., \& Scuderi, S. 2001, A\&A, 377, 123

Gray D.F. 1997, Nature, 385, 795

Gray D.F. 1998, Nature, 391, 153

Haisch K.E. Jr., Lada E.A., \& Lada C.J. 2001, ApJ, 553, L153

Halbwachs, J.-L., Mayor, M., \& Udry, S. 2003, in Extrasolar Planets: Today and Tomorrow, ed. C. Terquem, A. Lecavelier des Etangs, \& J.-P. Beaulieu, XIXth IAP Colloqium, in press

Halbwachs J.-L., Arenou F., Mayor M., Udry S., \& Queloz D. 2000, A\&A, 355, 573

Han I., Black D., \& Gatewood G. 2001, ApJ, 548, L57

Henry G.W., Donahue R.A., \& Baliunas S.L. 2002, ApJ, 577, L111

Henry, G.W., Marcy, G.W., Butler, R.P., \& Vogt, S.S. 2000, ApJ, 529, L41

Israelian G., Santos N.C., Mayor M., \& Rebolo R. 2003, A\&A, 405, 753

Israelian G., Santos N.C., Mayor M., \& Rebolo R. 2001, Nature, 411, 163

Jorissen, A., Mayor, M., \& Udry, S. 2001, A\&A, 379, 992

Konacki, M., Torres, G., Jha, Sa., Sasselov, D.D., 2002, Nature, 421, 507

Latham D.W., Stefanik R.P., Mazeh T., Mayor M., \& Burki G. 1989, Nature, 339,38

Laws, C., \& Gonzalez, G., Walker, K.M., Tyagi, S., Dodsworth, J., Snider, K., \& Suntzeff, N.B. 2003, AJ, 125, 2664

Laws, C., Gonzalez, G. 2001, ApJ, 553, 405

Masset F., \& Papaloizou, J. 2003, ApJ, 588, 494

Mayer, L., Quinn, T., Wadsley, J., \& Stadel, J. 2002, Science, 298, 1756

Mayor, M. 2003a, in Extrasolar Planets: Today and Tomorrow, ed. C. Terquem, A. Lecavelier des Etangs, \& J.-P. Beaulieu, XIXth IAP Colloqium, in press

Mayor, M., Udry, S., Naef, D., Pepe, F., Queloz, D., Santos, N.C., \& Burnet, M. 2003b, A\&A, in press

Mayor, M., \& Santos, N.C. 2002, in The Origins of Stars and Planets: the VLT View, Alves, J., \& McCaughrean M., ESO Astrophysics Symposia, Springer

Mayor, M., \& Udry, S. 2000 in APS Conf. Ser. Vol. 219, Disks, Planetesimals, and Planets, ed. F. Garzón, C. Euroa, D. de Winter, \& T. Mahoney, 441-453

Mayor, M., \& Queloz, D. 1995, Nature, 378, 355

Mazeh, T., \& Zucker, S. 2003, ApJ, 590, L115

Mazeh, T., \& Shaham, J. 1979, A\&A, 77, 145 
Murray, N., Paskiwitz, M., \& Holman, M. 2002, ApJ, 565, 608

Murray, N., Hansen, B., Holman, M., \& Tremaine, S. 1998, Science, 279, 69

Naef, D., Latham, D. W., Mayor, M., Mazeh, T., Beuzit, J. L., Drukier, G. A., Perrier-Bellet, C., Queloz, D., Sivan, J. P., Torres, G., Udry, S., \& Zucker, S. 2001, A\&A, 279, 69

Paulson D.B., Saar S.H., Cochran W.D., \& Hatzes A.P., 2002, AJ 124, 572

Pepe, F., Mayor, M., Rupprecht, G., et al. 2002, The ESO Messenger, 110, 9

Pinsonneault, M. H., DePoy, D. L., \& Coffee, M. 2001, ApJ, 556, L59

Pollack, J.B., Hubickyj, O., Bodenheimer, P., Lissauer, J.J., Podolak, M., \& Greenzweig, Y. 1996, Icarus, 124, 62

Pourbaix, D. 2001, A\&A, 369, L22

Pourbaix D., \& Arenou F. 2001, A\&A, 372, 935

Queloz D., Henry G.W., Sivan J.P., Baliunas, S.L., Beuzit, J.L., Donahue, R.A., Mayor, M., Naef, D., Perrier, C., \& Udry, S. 2001, A\&A, 379, 279

Rasio, F.A., \& Ford, E.B. 1996, Science, 274, 954

Reid I.N. 2002, PASP 114, 306

Sadakane, K., Ohkubo, M., Takada, Y., Sato, B., Kambe, E., \& Aoki, W. 2002, PASJ, 54, 911

Saar, S.H., Butler, R.P., \& Marcy, G.W. 1998, ApJ, 498, L153

Saar, S.H., \& Donahue, R.A. 1997, ApJ, 485, 319

Santos, N.C., Israelian, G., Mayor, M., Rebolo, R., \& Udry, S. 2003a, A\&A, 398,363

Santos, N.C., Udry, S., Mayor, M., Naef, D., Pepe, F., Queloz, D., Burki, G., Cramer, N., \& Nicolet, B. 2003b, A\&A, 406, 373

Santos, N.C., Mayor, M., Naef, D., Pepe, F., Queloz, D., Udry, S., Burnet, M., Clausen, J.V., Helt, B.E., Olsen, E.H., \& Pritchard, J.D. 2002, A\&A, 392,215

Santos, N.C., Israelian, G., \& Mayor, M. 2001, A\&A, 373, 1019

Santos, N.C., Mayor, M., Naef, D., Pepe, F., Queloz, D., Udry, S., Burnet, M., \& Revaz, Y. 2000a, A\&A, 356, 599

Santos N.C., Mayor M., Naef D., Pepe F., Queloz D., Udry S., \& Blecha A. 2000b, A\&A, 361, 265

Tinney, C.G., McCarthy, C., Jones, H.R.A., Butler, R.P., Carter, B.D., Marcy, G.W., \& Penny, A.J. 2002, MNRAS332, 759

Trilling, D., Lunine, J., \& Benz, W. 2002, A\&A, 394, 241

Udry, S., Mayor, M., Naef, D., Pepe, F., Queloz, D., Santos, N.C., \& Burnet, M. 2003b, A\&A, 407, 679

Udry, S., Mayor, M., \& Santos, N.C. 2003a, A\&A, 407, 369

Vogt S.S., Marcy G.W., Butler R.P., \& Apps K. 2000, ApJ, 536, 902

Ward, Wm. R. 1997, ApJ, 482, L211

Wolszczan, A., \& Frail, D.A. 1992, Nature, 355, 145

Zucker S., Mazeh T., Santos N.C., Udry S., \& Mayor M. 2003, A\&A, 404, 775

Zucker, S., \& Mazeh, T. 2002, ApJ, 568, L113 